\title{
Sistematización de los resultados obtenidos en los programas de vinculación con
} la sociedad que se ejecutan en los parques de la cuidad de Portoviejo. Systematization of the results obtained in the programs of connection with society that are carried out in the parks of the city of Portoviejo.

Sistematización de los resultados obtenidos en los programas de vinculación con la sociedad

\author{
Dr. Humberto Alvarado Medina ${ }^{(1)}$ \\ Lcda. Mirian Hernández Castro ${ }^{(2)}$ \\ Lcdo. Edwin Vinces Moreira ${ }^{(3)}$ \\ Lcda. Lucy Gioconda Quinteros Vargas ${ }^{(4)}$ \\ ${ }^{(1,2,3,4)}$ Instituto Superior Universitario Portoviejo
}

\section{Resumen}

La vinculación con la sociedad es un paso participativo, a través del cual los institutos de educación superior se plantean socialmente para actuar en la solución de los problemas de la comunidad, con el objetivo de evaluar el impacto que representa la carrera del tecnólogo de emergencias médicas y de enfermería, con esto realizamos un estudia de campo de enfermedades crónicas presentes en los usuarios de los parque para evaluar su presión arterial y su medición de peso y talla, con el fin de realizar evaluaciones, orientaciones, capacitaciones y recomendaciones, esta información nos ha servido para comprobar que la falta de información de los usuarios los hace vulnerables en la alimentación y hábitos saludables para los estilo de vida que presentan, por lo que pudimos comprobar que durante el cuarto trimestre del 2019, los parámetros de IMC, Y presión arterial disminuyeron en los usuarios que participaron en las diferentes capacitaciones y charlas educativa teniendo como positivo la gestión realizada en el marco del concepto de vinculación con la sociedad en los parques públicos del cantón Portoviejo de la provincia de Manabí. Con esto queremos resaltar la importancia que reviste el trabajo realizado por estudiantes de carrera de salud, en el proyecto de vinculación obteniendo valores positivos de mejoramiento de hábitos de vida de los ciudadanos donde se ejecuta el proyecto.

Palabras Clave: Comunidad, emergencias médicas, enfermedades crónicas, hábitos saludables.

\section{ABSTRACT}

The link with society is a participatory step, through which higher education institutes are socially raised to act in the solution of community problems, with the objective of assessing the impact of the career of the medical emergency technologist and nursing, with this we conduct a field study of chronic diseases present in park users 
to assess their blood pressure and their measurement of weight and height, in order to perform assessments, orientations, trainings and recommendations, this information gives us It has served to verify that the lack of information of the users makes them vulnerable in the diet and healthy habits for the lifestyle that they present, so we could verify that during the fourth quarter of 2019, the parameters of BMI, and blood pressure decreased in the users who participated in the different training and educational talks taking as positive the management carried out within the framework of the concept of linking with society in the public parks of the Portoviejo canton of the province of Manabí. With this we want to highlight the importance of the work carried out by students of health careers, in the linking project, obtaining positive values of improvement of life habits of the citizens where the project is executed.

Key Words: Community, medical emergencies, chronic diseases, healthy habits.

\section{INTRODUCCIÓN}

La sistematización desde la perspectiva científica, se basa en el modo de cómo abordar los objetos y fenómenos de forma integradora; prácticamente es una interacción que da como fruto, nuevas cualidades, o en este caso nuevas soluciones, a determinados problemas estudiados. La ciudad de Portoviejo al pasar de los años viene desarrollándose de manera en que la necesidad del área verde para recreación aumenta, es así que en el 2017 la municipalidad de la ciudad de Portoviejo inaugura los parques de La Rotonda y Las Vegas dándole así los espacios verdes tan necesarios a la comunidad, así mismo las necesidades de atención a emergencias crecen al mismo ritmo.

Durante los últimos años los ciudadanos residentes de la Ciudad de Portoviejo y usuarios de los parques. La Rotonda y Las vegas, se han visto afectados por el desconocimiento y desinformación, con respecto a una buena alimentación y hábitos de vida saludables, esto ha traído como consecuencia un aumento de las patologías crónicas como diabetes tipo II e hipertensión. En virtud de esta desinformación el Itsup ha desarrollado el proyecto zona protegida parques la Rotonda y Las Vegas, que busca generar conciencia en los ciudadanos para mejorar su calidad de vida.El paradigma de la investigación se basa en una intervención orientada hacia metodologías educativas más activas centradas en la corrección de hábitos de la población de estudio en el que se ejecuta la sistematización de los resultados obtenidos en los programas de vinculación con la sociedad que se ejecutan en los parques de la cuidad de Portoviejo. La hipótesis de partida consiste en mejorar los hábitos saludables en las personas que fueron participes de la toma de peso y talla en los parques donde se llevó a cabo la vinculación dejando claro que indirectamente se beneficiara a las familias del cantón; ya que estos son voceros para el resto de los integrantes. Objetivo general, Clasificar los resultados obtenidos en los programas de vinculación con la sociedad que se ejecutan en los parques de la cuidad de Portoviejo provincia Manabí 
durante el periodo de septiembre a diciembre de 2019. Según el estudio realizado se logró evidenciar a los usuarios de los Parques La Rotonda y Las Vegas, se logró investigar los niveles de vida y hábitos lo cual se obtuvieron mejores resultados con respecto a los valores iniciales, referentes a signos vitales y peso, con el fin de lograr los beneficios que se obtienen de estas condiciones, mejorando sus hábitos de vida y alimentario.El Ministerio de Salud Pública, asumiendo su rol y su competencia de autoridad sanitaria nacional, entidad a la que le corresponde el ejercicio de las funciones de rectoría en salud, posee programas que son participes de fomentar hábitos saludables en la población en general, forman clubes de personas que presentan enfermedades crónicas las mismas que son prevenibles mediante la asistencia estas actividades desarrolladas en cada área verde de nuestro cantón. "El entorno doméstico influye en la satisfacción de las necesidades básicas del individuo de estudio y de todo el grupo familiar, ya que tanto puede ser un factor protector de la salud como un factor de riesgo para la misma. Disponer de información sobre el entorno inmediato de la familia permite identificar las dificultades y los recursos que son de utilidad para la planificación de la atención a la familia” (Calero Muñoz \& Gloria, TRATADO DE MEDICINA DE FAMILIA Y COMUNITARIA, 2012, pág. 145). Valorar el entorno familiar basada en la satisfacción de las necesidades básicas de nutrición:

1. Hábitos y estados nutricionales.

2. Hábitos sociales respecto la comida.

3. Facilidad para adquirir y preparar los alimentos. Saez \& Font, (2002) Afirma: "forma de vida" refiere a los aspectos más objetivos (conductas) o como una "forma de entender la vida" si se hace referente a cognitivos y psicológicos (actitudes)". (Saez \& Font, 2002, pág. 48) Párr: $7^{7}$ El estilo de vida denota combinaciones de conductas que ocurren consistentemente. De hecho, se considera al estilo de vida como un modo de vida que abarca un amplio rango de conductas organizadas de una forma coherente en respuesta a las diferentes situaciones vitales de cada persona o grupo.

\section{El Sedentarismo}

El estilo de vida sedentario, que tantas complicaciones de salud causa durante toda la vida, la insuficiencia de ejercicios físicos que hace que los músculos que trabajan de forma insuficiente disminuyan la funcionabilidad de los órganos, a su vez se provocan alteraciones metabólicas de las grasas. El sedentarismo se relaciona con la reducción de la esperanza de vida y la pérdida del validismo, al influir en el desarrollo de enfermedades del aparato locomotor y de sostén. Entre estas tenemos:

- La cardiopatía.

- Enfermedades cerebro vasculares

- Aterosclerosis periférica.

- Obesidad.

- Diabetes mellitus. 
- Hipertensión arterial.

- Artritis, artrosis, radiculitis y dolores lumbares.

Entre las medidas que previenen este factor de riesgo está: realizar caminatas al día de $3 \mathrm{Km}$ o trotes de $2 \mathrm{~km}$, lo que puede significar disminución del peso corporal y colaborar al control de la obesidad, pues esta es una de las consecuencias del sedentarismo. La obesidad ha dejado de ser un problema real de la estética de la mujer y del hombre para convertirse en un verdadero problema de salud. Aunque la obesidad no es un fenómeno exclusivo de la época moderna, se ha venido transformando en una verdadera pandemia que alcanza a niños y jóvenes. Evaluación del estado nutricional "Será la acción y efecto de estimar, apreciar y calcular la condición en la que se halle un individuo según las modificaciones nutricionales que se hayan podido producir.

\section{MATERIALES Y MÉTODO}

Se realizó un estudio trasversal, descriptiva, ya que se basó en describir los factores de las variables involucradas en sus medidas antropométricas y signos vitales. Por su enfoque es un estudio mixto, porque se establecen relaciones entre variables cuantitativas y cualitativas, según el método utilizado es observacional, porque se describieron los resultados tal y como se dieron. Se revisaron los datos estadísticos de los usuarios que acudieron a los parques La Rotonda y Las vegas para la toma de signos vitales y peso la muestra de estudio fueron de 109 usuarios en el cuarto periodo del año 2019. Se realizó una estrategia de búsqueda basado en la recolección de datos de morbilidad obtenidos durante la ejecución del proyecto de vinculación con la comunidad que realizaron los estudiantes del Itsup y docentes durante las horas de cobertura en los parques La Rotonda y Las Vegas respectivamente.

\section{Técnicas e instrumentos de recolección de datos}

Entrevista semiestructurada con los participantes que acuden a diario a las horas de gimnasia en los distintos parques.

Observación Indirecta. Se llevará a cabo en el equipo de trabajo con el afán de motivar al entorno a corregir o mejorar los hábitos saludables.

\section{Técnicas de procesamiento y análisis de datos.}

El procesamiento de la información, permite ordenar, realizar gráficos en ciertos tipos de investigaciones e interpretar toda la información con base a los planteamientos teóricos, sustento del estudio realizado. 


\section{Resultados}

Se identificó las patológicas crónicas presentes en los usuarios que acudían a los parques por medio de la toma de signos y capacitación mediante charlas educativas a la comunidad afectada con eso se analizó los resultados obtenidos de los usuarios lo que arrojaron que hubo un mejoramiento es su estilo de vida y salud.

\begin{tabular}{|c|c|c|c|c|c|c|c|}
\hline Nombre & Apellido & Edad & $P A$ & $F R$ & Pulso & Peso & Talla \\
\hline Adrián & Cedeño & 19 & $120 / 80$ & 20 & 70 & 64.4 & 1.63 \\
\hline Alexandra & Quijije Cedeño & 59 & $130 / 80$ & 17 & 100 & 85 & 1.85 \\
\hline Alexandra & Mejia & 53 & $135 / 78$ & 20 & 66 & & \\
\hline Angelica & Loor & 60 & $111 / 76$ & 20 & 70 & & \\
\hline Angelica & Loor & 60 & $120 / 70$ & 20 & 70 & 66.4 & \\
\hline Antonella & $\begin{array}{l}\text { Vélez } \\
\text { Solórzano }\end{array}$ & 45 & $120 / 70$ & 18 & 80 & 80 & 1.87 \\
\hline Antonio & Vera Cusme & 59 & $130 / 80$ & 19 & 100 & 90 & 1.83 \\
\hline Benito & Solórzano $G$ & 63 & $130 / 80$ & 16 & 85 & 102 & 1.78 \\
\hline Betsi & Mejin & 53 & $130 / 80$ & 20 & 66 & 83.1 & \\
\hline Carlos & Chávez & 57 & $209 / 122$ & 22 & 87 & 85.4 & 1.69 \\
\hline Carlos & Cedeño & 65 & $106 / 67$ & 20 & 81 & 87.7 & \\
\hline Carlos & Cedeño & 65 & $113 / 71$ & 20 & 48 & 87.3 & 1.64 \\
\hline Cristhian & Quijije & 22 & $140 / 90$ & 20 & 120 & 70 & 1.85 \\
\hline Daniel & Andrade & 63 & $130 / 80$ & 17 & 98 & 75 & 1.70 \\
\hline Daniel & Zamora & 39 & $120 / 80$ & 20 & 100 & 85 & 1.70 \\
\hline Didimo & Pérez. & 57 & $126 / 103$ & 22 & 83 & & \\
\hline
\end{tabular}


Revista Sinapsis. Vol. 2, Nro 20, diciembre de 2021, ISSN 1390 - 9770

\begin{tabular}{|c|c|c|c|c|c|c|c|}
\hline Didimo & Pérez & 57 & $132 / 67$ & 22 & 72 & 115 & 1.72 \\
\hline Didimo & Pérez & 57 & $130 / 66$ & 20 & 88 & 111 & 1.72 \\
\hline Didimo & Pérez & 57 & $110 / 70$ & & & & 1.72 \\
\hline Didimo & Pérez & 57 & $120 / 80$ & 20 & 74 & 114 & \\
\hline Didimo & Pérez & 57 & $131 / 78$ & 18 & 68 & 111.7 & 1.72 \\
\hline Didimo & Pérez & 57 & $150 / 90$ & & & 110 & 1.72 \\
\hline Dora & Cevallos & 60 & $110 / 70$ & & & 61.4 & \\
\hline Dora & Menendez & 60 & $104 / 68$ & 22 & 54 & 61.2 & 1.49 \\
\hline Eduardo & Quijije & 67 & $150 / 79$ & 18 & 93 & 55.3 & 1.68 \\
\hline Eduardo & Quijije & 67 & $146 / 94$ & 16 & & 72.1 & 1.68 \\
\hline Eduardo & Quijije & 67 & $158 / 85$ & 18 & & 71.1 & 1.68 \\
\hline Edwin & Reyes Salazar & 60 & $120 / 80$ & 15 & 70 & 99 & 1.80 \\
\hline Erilda & Vera & 63 & $136 / 71$ & 20 & & 74.2 & 1.56 \\
\hline Erminia & Saltos & 67 & $100 / 50$ & 20 & 80 & 52.0 & 1.50 \\
\hline Erminia & Saltos & 67 & $100 / 50$ & 18 & & 51.8 & 1.51 \\
\hline Ernesto & Zambrano & 61 & $140 / 90$ & 23 & 110 & 80 & 1.95 \\
\hline Estelita & $\begin{array}{l}\text { Ballejos } \\
\text { Moreira }\end{array}$ & 73 & $130 / 90$ & 20 & 98 & 89 & 1.70 \\
\hline Ester & Barrezueta & 45 & $128 / 80$ & 19 & & 77.6 & 1.30 \\
\hline Eulalio & Menendez & 66 & $110 / 80$ & 20 & & 64.6 & \\
\hline Eulalio & Menendez & 66 & $142 / 74$ & 20 & 69 & 65.8 & 1.56 \\
\hline
\end{tabular}


Revista Sinapsis. Vol. 2, Nro 20, diciembre de 2021, ISSN 1390 - 9770

\begin{tabular}{|c|c|c|c|c|c|c|c|}
\hline Florcita & Panchón & 65 & $130 / 80$ & 17 & 90 & 85 & 1.65 \\
\hline Fortunato & $\begin{array}{l}\text { Cevallos } \\
\text { Ponce }\end{array}$ & 64 & $130 / 80$ & 18 & 100 & 79 & 1.78 \\
\hline Fredy & Moreira & 35 & $120 / 70$ & 20 & 90 & 95 & 1.72 \\
\hline Gabriela & Ruiz & 38 & $140 / 72$ & 20 & 70 & 54.4 & 1.67 \\
\hline Héctor & Murillo & 38 & $110 / 80$ & 19 & 98 & 92 & 1.78 \\
\hline Idalda & Vera & 63 & $100 / 60$ & & & 74.2 & 1.56 \\
\hline Idaura & Vinces & 52 & $110 / 80$ & 20 & & 92.6 & 1.58 \\
\hline Iralda & Vera & 63 & $110 / 77$ & 18 & 90 & 74.0 & 1.53 \\
\hline Iralda & Vera & 63 & $118 / 80$ & 20 & 89 & 75.2 & 1.56 \\
\hline Iván & Moreira & 45 & $120 / 70$ & 16 & 110 & 78 & 1.80 \\
\hline Jaime & $\begin{array}{l}\text { Sancan } \\
\text { Figueroa }\end{array}$ & 60 & $140 / 90$ & 22 & 130 & 16 & 1.80 \\
\hline Jaqueline & Mendoza & 54 & $119 / 74$ & 20 & 89 & 80.4 & 1.56 \\
\hline Jerónimo & $\begin{array}{l}\text { Alcívar } \\
\text { Menéndez }\end{array}$ & 65 & $140 / 70$ & 23 & 120 & 80 & 1.85 \\
\hline Jorge & $\begin{array}{l}\text { Barreto } \\
\text { Zambrano }\end{array}$ & 60 & $140 / 80$ & 22 & 100 & 85 & 1.65 \\
\hline Jose & $\begin{array}{l}\text { Navia } \\
\text { Zambrano }\end{array}$ & 61 & $140 / 90$ & 25 & 110 & 80 & 1.95 \\
\hline Jose & Sabando & 62 & $139 / 76$ & 20 & 66 & 82.9 & \\
\hline Jose & Macias & 41 & $124 / 70$ & 20 & 80 & & \\
\hline
\end{tabular}


Revista Sinapsis. Vol. 2, Nro 20, diciembre de 2021, ISSN 1390 - 9770

\begin{tabular}{|c|c|c|c|c|c|c|c|}
\hline José & $\begin{array}{l}\text { Macas } \\
\text { Calderon }\end{array}$ & 23 & $150 / 90$ & 25 & 125 & 92 & 1.75 \\
\hline Juan & Cedeño & 43 & $130 / 90$ & & & & 1.65 \\
\hline Juan Carlos & Cedeño & 43 & $140 / 80$ & 20 & 90 & 109.4 & 1.65 \\
\hline Juan carlos & Cedeño & 43 & $130 / 90$ & 22 & 85 & 109.6 & 1.63 \\
\hline Juan Carlos & Cedeño & 43 & $130 / 90$ & & & 109.2 & 1.63 \\
\hline Karen & Molina & 19 & $130 / 90$ & 22 & 110 & 75 & 1.70 \\
\hline Katy & Mendoza & 42 & $117 / 78$ & 20 & 74 & 77.1 & 1.63 \\
\hline Klever & Pinargote & 52 & $130 / 80$ & 20 & 82 & 69.5 & 1.67 \\
\hline Luci & Velez. & 49 & $100 / 80$ & & & 61.3 & \\
\hline Lueta & $\begin{array}{l}\text { Macías } \\
\text { Berduga }\end{array}$ & 65 & $120 / 90$ & 17 & 70 & 101 & 1.67 \\
\hline Luis & Roldan & 64 & $160 / 88$ & 20 & 66 & & \\
\hline Luis & Salazar & 60 & $128 / 82$ & 20 & 82 & 96.3 & 1.67 \\
\hline Manuel & Bravo & 60 & $120 / 70$ & 17 & 90 & 85 & 1.80 \\
\hline Maria & Garcia & 39 & $108 / 60$ & 20 & 70 & 73.0 & 7.59 \\
\hline Maria & Pinargote & 44 & $144 / 83$ & 20 & 87 & 61.6 & 1.54 \\
\hline Maria & Garcia & 39 & $97 / 89$ & 19 & 76 & 72.7 & 1.56 \\
\hline Maria & Garcia & 39 & $113 / 64$ & 20 & 69 & 73.0 & 1.59 \\
\hline Maria & Fernandez & 11 & $97 / 58$ & 20 & 97 & 37 & 1.39 \\
\hline Maribel & Moreira & 34 & $11 / 63$ & 20 & 88 & 79.4 & 1.67 \\
\hline
\end{tabular}


Revista Sinapsis. Vol. 2, Nro 20, diciembre de 2021, ISSN 1390 - 9770

\begin{tabular}{|c|c|c|c|c|c|c|c|}
\hline Maritza & Romero & 61 & $110 / 80$ & 20 & 70 & 95.6 & 1.60 \\
\hline Marjorie & Pinargote & 44 & $120 / 80$ & 18 & 86 & 68.8 & 1.60 \\
\hline Mercedez & Moreira & 59 & $123 / 78$ & 18 & & 61.2 & 1.57 \\
\hline Miguel & Suarez & 39 & $116 / 66$ & 20 & 57 & & \\
\hline Miguel & Suarez & 67 & $150 / 82$ & & & 77.1 & \\
\hline Miguel & Suarez & 67 & $139 / 70$ & 20 & 57 & 76.2 & \\
\hline Miguel & Suarez & 67 & $130 / 90$ & 18 & & 75.5 & 1.65 \\
\hline Miguel & Suarez & 67 & $130 / 70$ & 20 & & 75.8 & 1.65 \\
\hline Modesta & Vélez. & 51 & $119 / 70$ & 20 & 78 & 74.5 & 1.56 \\
\hline Nelly & $\begin{array}{l}\text { Vélez } \\
\text { Solórzano }\end{array}$ & 57 & $140 / 90$ & 20 & 110 & 92 & 1.79 \\
\hline Nevi & Moreira & 59 & $197 / 118$ & 20 & 83 & & \\
\hline Nevi & Moreira & 59 & $170 / 80$ & 16 & 79 & 71.4 & 1.63 \\
\hline Nevi & Moreira & & $150 / 90$ & & & & \\
\hline Nevi & Moreira & 59 & $100 / 116$ & 16 & 82 & 71.1 & 1.63 \\
\hline Nevi & Moreira & 59 & $175 / 104$ & 20 & & 71.4 & 1.68 \\
\hline Olinda & Barbaran & 75 & $120 / 80$ & 16 & 100 & 90 & 1.77 \\
\hline Orlando & Alcivar & 61 & $140 / 70$ & 22 & 70 & 45.8 & 1.72 \\
\hline Pablo & Cevallos & 35 & $150 / 100$ & 23 & 130 & 80 & 1.81 \\
\hline Paulette & Sánchez & 45 & $120 / 80$ & 17 & 90 & 93 & 1.86 \\
\hline Ramon & Chavez & 63 & $133 / 86$ & 18 & & 103.1 & 1.72 \\
\hline
\end{tabular}


Revista Sinapsis. Vol. 2, Nro 20, diciembre de 2021, ISSN 1390 - 9770

\begin{tabular}{|c|c|c|c|c|c|c|c|}
\hline Ramón & Chávez & 67 & $120 / 80$ & 18 & 87 & 101.8 & 1.72 \\
\hline Redis & $\begin{array}{l}\text { Valencia } \\
\text { Cobeña }\end{array}$ & 48 & $130 / 70$ & 17 & 100 & 65 & 1.79 \\
\hline Roque & $\begin{array}{l}\text { Macias } \\
\text { Parraga }\end{array}$ & 53 & $110 / 70$ & 16 & 80 & 97 & 1.82 \\
\hline Rosario & Meza & 57 & $98 / 63$ & 20 & 77 & 64.3 & 1.63 \\
\hline Rosario & Meza & 57 & $85 / 57$ & 20 & 78 & 65.2 & 1.63 \\
\hline Rosario & Meza & 57 & $98 / 61$ & 20 & 75 & 65.3 & 1.63 \\
\hline Rosario & Meza & 57 & $100 / 61$ & 20 & 78 & 65.4 & 1.63 \\
\hline Rosario & Meza & 57 & $91 / 56$ & 20 & 83 & 67.1 & 1.63 \\
\hline Rosario & Meza & 57 & $96 / 63$ & 20 & 70 & 65.6 & 1.63 \\
\hline Rosario & Meza & 57 & $97 / 74$ & 22 & & 64.7 & 1.63 \\
\hline Ruben & Sanchez & 67 & $130 / 70$ & 18 & 72 & 55.5 & 1.50 \\
\hline Ruben & Sanchez & 63 & $122 / 60$ & 20 & 68 & & \\
\hline Ruth & Vélez. & 41 & $130 / 90$ & 19 & 110 & 78 & 1.68 \\
\hline Susana & Sánchez & 53 & $110 / 70$ & 22 & 90 & 79.7 & 1.65 \\
\hline Vicente & Bravo Tuares & 55 & $130 / 60$ & 18 & 75 & 87 & 1.79 \\
\hline Víctor & Vélez & 65 & $130 / 80$ & 20 & 100 & 79 & 1.92 \\
\hline Walter & Pincay & 30 & $120 / 70$ & 20 & 80 & 90 & 1.75 \\
\hline William & Arteaga & 57 & $134 / 75$ & 20 & 86 & 85.2 & 1.74 \\
\hline Willian & Arteaga & 57 & $115 / 80$ & 20 & 71 & & \\
\hline
\end{tabular}


Revista Sinapsis. Vol. 2, Nro 20, diciembre de 2021, ISSN 1390 - 9770

\begin{tabular}{|l|l|l|l|l|l|l|l|}
\hline Yani & Zambrano & 40 & $120 / 70$ & 19 & 78 & 90 & 176 \\
\hline Zoila & Navia & 38 & $130 / 70$ & 18 & 85 & 95 & 1.75 \\
\hline
\end{tabular}

\section{Discusión (o análisis de resultados)}

En Ecuador, de cada 100.000 personas, 1.373 tienen problemas de hipertensión, así lo afirma el informe 2012 de la Organización Mundial de la Salud (OMS). Anualmente esta enfermedad causa la muerte a 9,4 millones de personas en el mundo. En el año 2011 a nivel nacional se presentan 10.429 casos con enfermedades hipertensivas, siendo la hipertensión esencial (primaria) la que muestra el mayor número de casos, con 8.653. Del total de registros, el 58,14\% corresponde a los hombres y el 41,86\% a mujeres. Al analizar por grupos de edad, los más vulnerables a sufrir hipertensión arterial son los adultos mayores, que representan el 52,39\%.

Se distinguen entre los resultados de mayor relevancia a 4 hombres y una mujer que oscilan en edades de 57 (2 personas), 59 (1 persona), 67 (2 personas), los cuales reflejaban patologías crónicas o incapacitantes son aquellas que comúnmente se adquieren por medio de estilos de vidas inapropiados, aunque siempre hay que considerar que existen factores genéticos de naturaleza hereditaria que concierne a estas condiciones.

"La hipertensión arterial (HAS) se define como la elevación permanente de la presión en el sistema arterial. En la actualidad estos límites se encuentran en los 140 mmHg para la sistólica y 90 para la diastólica" (Robledo, 2014, pág. 264) Cap.:25

"Presión arterial se define por la presencia de los valores superiores a la normalidad de manera persistente. La raza afroamericana tiene una mayor prevalencia del doble en relación a la blanca" (Jánez \& Padilla, 2013, pág. 133)

"La OMS define a la actividad problema de nivel público en las personas, el déficit de actividad física se relaciona con el aumento de la tensión arterial. Se conoce que ejercer el cuerpo en forma continua disminuye la HTA en habitantes con presión normal o alta, tanto en varones como en mujeres (SAHA, 2011)

Las áreas verdes urbanas poseen un elevado potencial de generación de beneficios para sus habitantes tanto directos como indirectos, que pueden ayudar a solucionar muchos de los problemas relacionados con las enfermedades prevenibles mediante hábitos saludables. Estas áreas mejoran la calidad del aire, debido a la vegetación, tienen impactos positivos sobre la salud física, como puede ser la disminución de las enfermedades respiratorias y crónico degenerativas, reducen el estrés al contribuir a un ambiente estéticamente placentero y relajante; ya es conocido en diferentes estudios, la existencia entre el hábito de fumar, el consumo de alcohol, el sedentarismo, la ansiedad y los malos hábitos alimenticios; todo acumulado por el stress laboral. 


\section{Conclusión}

El diseño y gestión de zonas verdes, tanto urbanas como periurbanas, es una herramienta versátil y eficaz en la lucha contra los problemas medioambientales de las ciudades y en la búsqueda de su sostenibilidad, ya que los parques y jardines poseen un elevado potencial de generación de beneficios.

La sistematización de resultados que se obtuvieron en la vinculación ejecutada en los parques, notaremos la relación que existe entre los hábitos saludables y la actividad física en lugares abierto rodeados de áreas verdes. El desapego a programas que incentiven hábitos saludables es el principal causante del sobrepeso y obesidad en él incide el desequilibrio entre la ingesta y el gasto energético.

\section{Referencias Bibliográficas}

Calero Muñoz, S. C., \& Gloria. (2012). TRATADO DE MEDICINA DE FAMILIA Y COMUNITARIA . españa: Panamericana.

Saez, S., \& Font, P. (2002). PREVENCION Y EDUCACION PARA LA SALUD . Mexico: MILENIO .

Robledo, D. C. (2014). Geriatría (Tercera Edición ed.). (D. R. Vela, Ed.) Mexico , Mexico : El Manual Moderno.

SAHA. (2011). Hipertensión y modificaciones en el estilo de vida. Obtenido de Hipertensión y modificaciones en el estilo de vida: http://www.saha.org.ar/hipertension-y-modificaciones-en-el-estilo-de-vida.php

Yanez, P. A., \& Padilla, D. M. (2008). Guia clínica Geronto-geriatricas de atención . Quito-Ecuador: OMS 